\title{
Large ovarian cyst with torsion
}

\author{
Moses Mhayamaguru ${ }^{1} \cdot$ Edward Haynes Bruno $^{2} \cdot$ Suzanne Michelle Rhodes $^{1} \cdot$ \\ Richard Amini ${ }^{1}$
}

Received: 4 August 2016/Accepted: 9 August 2016/Published online: 16 August 2016

(C) SIMI 2016

\section{Patient presentation}

A 20-year-old, otherwise healthy, G1P1 woman presents to the emergency department complaining of sudden onset right upper quadrant (RUQ) abdominal pain, for the prior $3 \mathrm{~h}$, resolving prior to examination. The pain was associated with nausea and vomiting. For the past month, she had experienced sharp, interment, RUQ pain of fluctuating severity. She denied fevers, chills, dysuria, vaginal bleeding, discharge, or diarrhea. The physical examination was notable for blood pressure of $152 / 92 \mathrm{mmHg}$ and BMI of 43.9.

\section{Discussion}

The workup demonstrated a negative pregnancy test and a mildly elevated AST/ALT. The emergency physician ordered RUQ and abdominal ultrasounds, which demonstrated a normal gallbladder but localized a $13.6 \mathrm{~cm}$ anechoic cystic structure at the level of the umbilicus. Within the cyst was a daughter cyst, without significant bloodflow (Fig. 1). An MRI of the pelvis revealed a $14.5 \mathrm{~cm}$ cyst arising from an edematous right ovary. The presence of an internal daughter cyst was confirmed (Fig. 2). Given the history of intermittent abdominal pain and the imaging findings, concern arose for intermittent ovarian torsion. Laparoscopic right salpingo-oophorectomy was performed. The surgical pathology revealed a serous cystadenoma with edema and hemorrhage consistent with torsion.

Serous cystadenomas are thin-walled, usually unilateral, uni-/or multilocular cysts, ranging from 5 to over $20 \mathrm{~cm}$, and are the most common benign ovarian neoplasms in women of reproductive age. Torsion is a complication of ovarian tumors, with an incidence $6.9 \%$ of all ovarian tumors, with an average patient age of 29.8 (range 11-57), that occurs more commonly in pregnant than non-pregnant women with ovarian tumors (22.7 vs $6.1 \%$ ) [1]. Benign ovarian neoplasms are 12.9 times more likely to undergo torsion (CI $95 \%, 10.2-15.9)$ - possibly related to the tendency of malignancies to adhere to local structures [2]. The presentation of ovarian torsion classically includes acute onset lower abdominal pain and a palpable adnexal mass; nausea and vomiting occur in $70 \%$ of patients [3]. Ultrasound is a commonly used imaging modality in evaluating acute pelvic pain; cystic etiologies of torsion are easily visualized $[3,4]$.

Richard Amini

richardamini@gmail.com

1 Department of Emergency Medicine, University of Arizona, PO Box 245057, Tucson, AZ 85724-5057, USA

2 College of Medicine, University of Arizona, Tucson, AZ, USA 


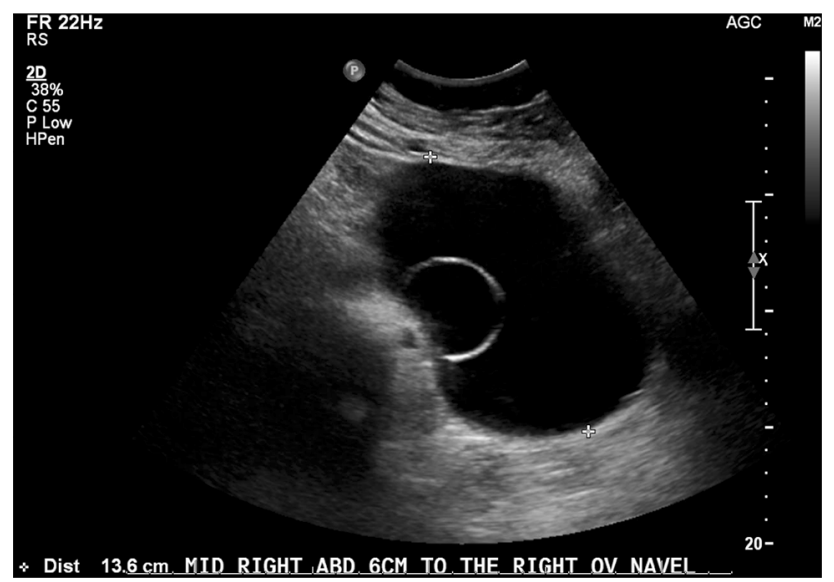

Fig. 1 Abdominal ultrasound demonstrating a $13.6 \mathrm{~cm}$ anechoic cystic structure at the level of the umbilicus. Within the cyst was a second cystic structure

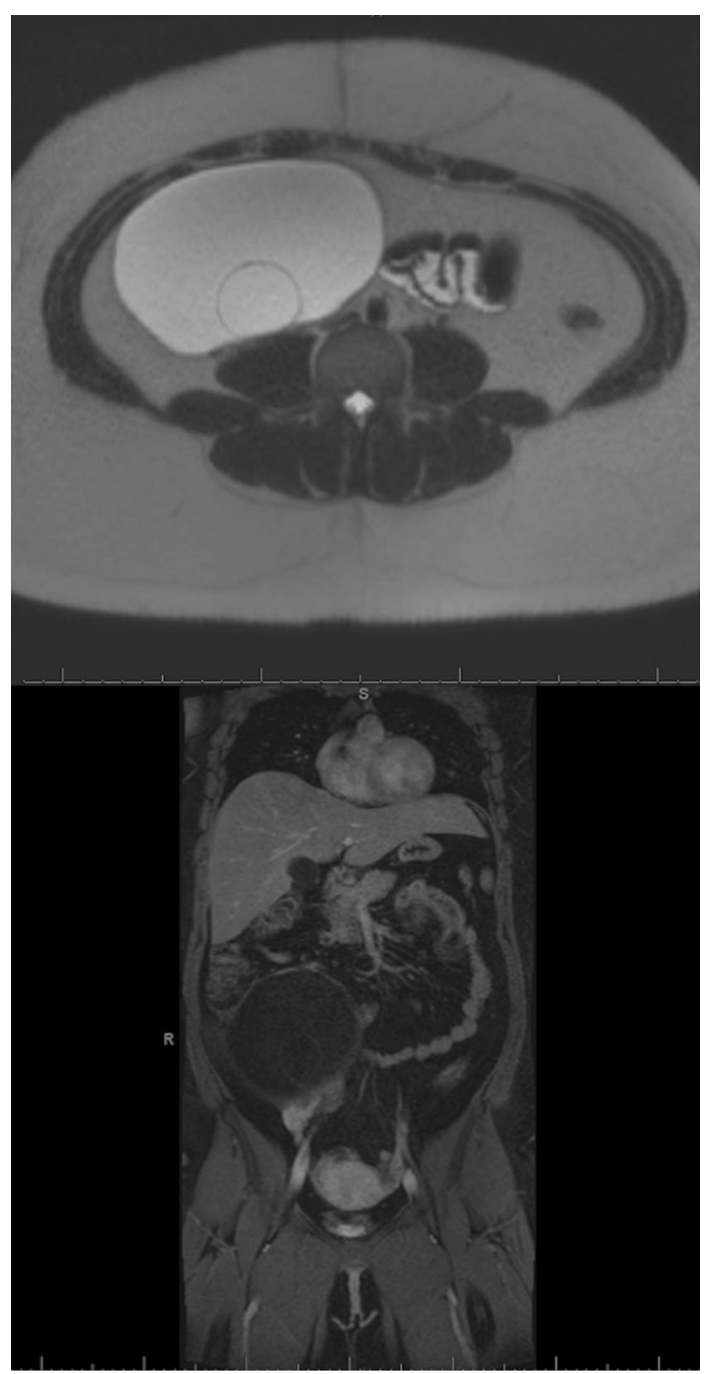

Fig. 2 MRI of the pelvis revealed a $14.5 \mathrm{~cm}$ cyst arising from an edematous right ovary. The presence of an internal daughter cyst was confirmed

\section{Compliance with ethical standards}

Conflict of interest The authors declare that they have no conflict of interest.

Statement of human and animal rights All procedures performed in studies involving human participants were in accordance with the ethical standards of the institutional and/or national research committee and with the 1964 Helsinki declaration and its later amendments or comparable ethical standards.

Informed consent Informed consent was obtained from all individual participants included in the study.

\section{References}

1. Lee CH, Raman S, Sivanesaratnam V (1989) Torsion of ovarian tumors: a clinicopathological study. Int $\mathrm{J}$ Gynaecol Obstet 28(1):21-25

2. Sommerville M, Grimes DA, Koonings PP, Campbell K (1991) Ovarian neoplasms and the risk of adnexal torsion. Am J Obstet Gynecol 164(2):577-578

3. Oelsner G, Shashar D (2006) Adnexal torsion. Clin Obstet Gynecol 49(3):459-463

4. Smorgick N, Maymon R (2014) Assessment of adnexal masses using ultrasound: a practical review. Int $\mathrm{J}$ Womens Health 6:857-863 